\title{
Piece-wise linear functions-based model predictive control of large-scale sewage systems
}

\section{Ocampo-Martinez V. Puig}

Institut de Robòtica i Informàtica Industrial (CSIC - UPC), Llorens i Artigas, 4-6, 08028 Barcelona, Spain E-mail: cocampo@iri.upc.edu

\begin{abstract}
In this study, model predictive control (MPC) of large-scale sewage systems is addressed, considering several inherent continuous/discrete phenomena (overflows in sewers and tanks) and elements (weirs) in the system. This fact results in distinct behaviour depending on the dynamic state (flow/volume) of the network. These behaviours cannot be neglected nor can be modelled by a pure linear representation. In order to take into account these phenomena and elements in the design of the control strategy, a modelling approach based on piece-wise linear functions (PWLF) is proposed and compared against a hybrid modelling approach previously suggested by the authors. Control performance results and associated computation times of the closed-loop scheme considering both modelling approaches are compared by using a real case study based on the Barcelona sewer network. Results have shown an important reduction in the computation time when the PWLF-based model is used, with an acceptable suboptimality level in the closed-loop system performance.
\end{abstract}

\section{Introduction}

Sewer networks are considered as complex large-scale systems since they are geographically distributed and interconnected with a hierarchical structure. Each subsystem is composed of a large number of elements with time-varying behaviour, exhibiting numerous operating modes and subject to dynamic changes because of external conditions (weather) and operational constraints.

Most cities around the world have sewage systems that combine sanitary and storm water flows within the same network. This is why these networks are known as combined sewage systems (CSS). During rain storms, wastewater flows can easily overload these CSS, thereby causing operators to dump the excess of water into the nearest receiver environment (rivers, streams or sea). This discharge to the environment, known as combined sewage overflow (CSO), contains biological and chemical contaminants creating a major environmental and public health hazard. Environmental protection agencies have started forcing municipalities to find solutions in order to avoid those $\mathrm{CSO}$ events. A possible solution to the $\mathrm{CSO}$ problem would be to enhance existing sewer infrastructure by increasing the capacity of the wastewater treatment plants (WWTP) and by building new underground retention tanks. But in order to take profit of these expensive infrastructures, it is also necessary a highly sophisticated real-time control (RTC) scheme, which ensures that high performance can be achieved and maintained under adverse meteorological conditions [1]. The advantage of RTC applied to sewer networks has been demonstrated by an important number of researchers during the last decades, see [2-5].

An RTC scheme in sewage systems might be 'local' (when flow regulation devices use only measurements taken at their specific locations) or 'global' (when control actions are computed taking into account real-time measurements all through the network). Since a sewer network as a complex system shows strong cross-relation between its elements, a global RTC is the proper strategy to manage and control this type of systems. The multivariable and large-scale nature of sewer networks have lead to the use of some 
variants of receding horizon control (RHC) - also referred as model predictive control (MPC) - as global control strategies $[2,5]$. In order to use RHC within a global RTC scheme of a sewage system, a model able to predict its future states over a prediction horizon taking into account a rain forecast is needed.

Sewer networks are systems with complex dynamics since water flows through sewers in open channel. When developing a control-oriented model, there is always a trade-off between model description accuracy and computational complexity. Several control-oriented modelling techniques presented in the literature deal with the global RTC of sewage systems, see $[5,6]$. In $[7,8]$, a conceptual linear model is used based on the assumption that a set of sewers in a catchment can be considered as a 'virtual tank'. The main reason to use a linear model is to preserve the convexity of the optimisation problems related to the RHC strategy. A similar approach can be found in an early reference on RHC applied to sewage systems [2]. However, there exist several inherent phenomena (overflows in sewers and tanks) and elements (weirs) in the system that result in distinct behaviour depending on the state (flow/volume) of the network. These discontinuous behaviours cannot be neglected nor can be modelled by a pure linear model. Additionally, the presence of intense precipitation causes that new flow paths appear. Thus, some flow paths are not always present in the sewer network and depend on its state and disturbances (rain). The description and analysis of these continuous/discrete dynamic behaviours on sewer networks have been previously reported by the authors [9]. In that work, it was also presented an hybrid modelling approach based on the 'mixed logical dynamical' (MLD) form - introduced in [10] - oriented to the design of RHC-based RTC scheme for large-scale sewage systems. However, it was shown that the inclusion of those discontinuous behaviours in the RHC problem increases the computation time of the control law. So, some relaxation in the modelling approach should be thought such that it can be considered within the RTC of large-scale sewer networks.

The aim of this paper is to propose an alternative modelling approach that represents the sewage system by using piece-wise linear functions (PWLF; in the sequel called PWLF-based model or simply PWLF model), following the ideas proposed by Schechter [11]. The purpose of this modelling approach is to reduce the complexity of the RHC problem by avoiding the logical variables introduced by the MLD system representation. The idea behind the PWLF-based modelling approach consists in having a description of the network using functions that, despite their discontinuous nature, are considered as quasi-convex [12], fact that might yield to the quasi-convexity of optimisation problems associated to the non-linear MPC strategy used for RTC of the sewage system [8]. In this way, the resultant optimisation problems do not include integer variables, what allows saving computation time.
The remainder of the paper is organised as follows. In Section 2, control-oriented modelling of sewer networks is revised. The issue of hybrid dynamics is presented and addressed using the proposed PWLF-based modelling approach. RTC scheme for sewage systems based on MPC strategy is addressed in Section 3, taking into account the modelling approach presented in the previous section. Section 4 presents the real case study based on the Barcelona sewer network used in this paper. This case study is used to show the computational improvements when implementing a predictive controller based on the modelling approach presented in Section 2 with respect to the hybrid approach suggested in [13]. Section 5 shows and discusses the control results obtained in several scenarios in the proposed case study. Finally, in Section 6 the conclusions are drawn.

\section{RTC-oriented modelling of large-scale sewer networks}

One of the most important stages on the design of RTC schemes for sewer networks, in the case of using a modelbased control technique as MPC, lies on the modelling task. This is because performance of model-based control techniques relies on model quality. So, in order to design an MPC-based RTC scheme with a proper performance, a system model with accuracy enough should be used but keeping complexity manageable. This section reviews the principles of the mathematical RTC-oriented modelling of sewer networks. Additionally, it shows how the proposed PWLF-based modelling framework is used to take into account the inherent hybrid behaviours in the different elements of the sewer network.

\subsection{Principles of mathematical RTC modelling of sewage systems}

Water flow in sewer pipes is open-channel, which corresponds to the flow of a certain fluid in a channel in which the fluid shares a free surface with an empty space above. The Saint-Venant equations, based on physical principles of mass conservation and energy, allow the accurate description of the open-channel flow in sewer pipes [14] and therefore also allow to have a detailed nonlinear description of the system behaviour. These equations are expressed as

$$
\begin{gathered}
\frac{\partial q_{x, t}}{\partial x}+\frac{\partial A_{x, t}}{\partial t}=0 \\
\frac{\partial q_{x, t}}{\partial t}+\frac{\partial}{\partial x}\left(\frac{q_{x, t}^{2}}{A_{x, t}}\right)+g A_{x, t} \frac{\partial L_{x, t}}{\partial x}-g A_{x, t}\left(I_{0}-I_{f}\right)=0
\end{gathered}
$$

where $q_{x, t}$ is the flow $\left(\mathrm{m}^{3} / \mathrm{s}\right), A_{x, t}$ is the cross-sectional area of the pipe $\left(\mathrm{m}^{2}\right), t$ is the time variable (s), $x$ is the spatial variable measured in the direction of the sewage flow $(\mathrm{m}), g$ is the gravity $\left(\mathrm{m} / \mathrm{s}^{2}\right), I_{0}$ is the sewer pipe slope (dimensionless), $I_{f}$ is the friction slope (dimensionless) and $L_{x, t}$ is the water 
level inside the sewer pipe (m). This pair of partialdifferential equations constitutes a non-linear hyperbolic system. For an arbitrary geometry of the sewer pipe, these equations lack of an analytical solution. Notice that these equations describe the system behaviour in high detail. However, such a level of detail is not useful for RTC implementation because of the complexity and the high computational cost of combining (1) and (2) with the RHC strategy.

Alternatively, several conceptual modelling techniques that deal with RTC of sewer networks have been presented in the literature, see $[5,6,15,16]$, among many others. The modelling approaches presented in this paper follow closely the mathematical modelling principles proposed in [2]. Here sewage system is divided into catchments and the set of pipes storage capacity belonging to each partition is modelled as a virtual tank. At any given time, the stored volumes represent the amount of water stored inside the sewer pipes associated with. The virtual tank volume is calculated through the mass balance of the stored volume, the inflows and outflows of the catchment measured using limnimetres and the input rain intensity measured using rain-gauges.

Using the virtual tank modelling principle and the mass balance conservation law, a sewer network can be decomposed and described by using the elementary models explained below and shown in Figs. 1 and 2, element by element and conforming a simple network, respectively. Other common sewage system elements such as pumping stations can be easily represented by using the mentioned
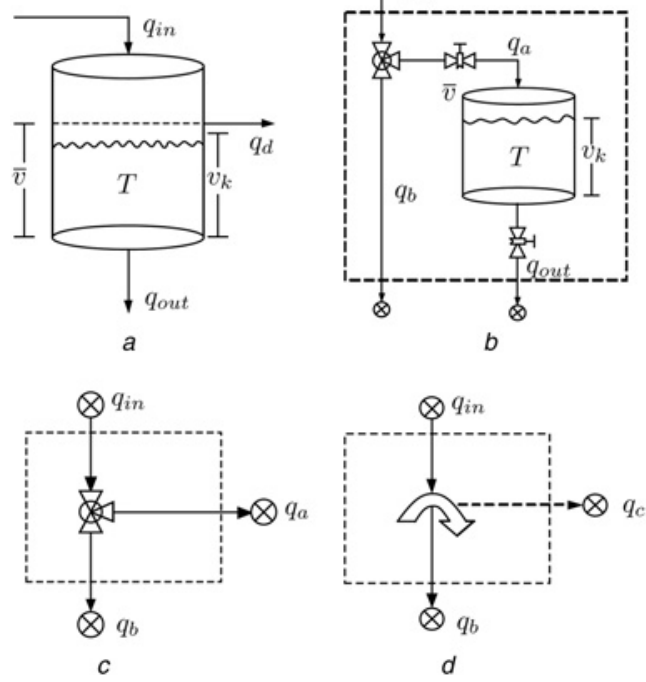

Figure 1 Conceptual schemes for sewer networks constitutive elements
a Virtual tank
$b$ Real tank
c Redirection gate
$d$ Sewer pipe or weir with single inflow

modelling principles but will be omitted here as they are not taken into account in the case study presented in this paper. Next, sewer network constitutive elements will be expressed including a conceptual scheme which will be not only used for describing its operation but also for explaining the mathematical relations and equations derived when using the PWLF-based modelling approach.

\subsection{PWLF-based modelling of sewer network constitutive elements}

As discussed in the introduction, sewer networks present several inherent hybrid behaviours that cannot be modelled using a pure linear model. In this paper, the modelling framework based on PWLFs is used to model such behaviours. More precisely, the proposed PWLF-based modelling methodology consists in using continuous and monotonic functions to represent expressions that contain logical conditions, which describe the weirs behaviour and overflow capability of reservoirs, respectively. Indeed, these phenomena involve the switching and discontinuous behaviours of the sewage system. The PWLF approach is though as an alternative to the use of a pure hybrid modelling approach, already proposed for the RTC of sewer networks [13].

The PWL functions used to model the discontinuous behaviours of sewer networks are defined as 'saturation' of a variable $x$ in a value $M$ (i.e. sat $(x, m)$ ), and 'dead zone' of the same variable $x$ starting in a value $M$ (i.e. $\operatorname{dzn}(x, M)$ ). Those functions are monotonic and continuous and might lead to a quasi-convex optimisation problem when formulating the MPC problem. According to [12], the global optimal solution of quasi-convex optimisation problems can be obtained by using a bisection method, which is logarithmic in time. This represents an advantage with respect to the mixed-integer linear problems resultant when using a pure hybrid approach based on MLD or piecewise affine (PWA) approaches. This type of models induces an exponential complexity given by the handling of Boolean variables and the discrete optimisation required.

2.2.1 Virtual and real tanks: These elements are used as storage devices. In the case of virtual tanks (see Fig. 1a), the mass balance of the stored volume, the tank inflows and outflows and the input rain intensity can be written as the difference equation with a sampling time $\Delta t$

$$
v_{i k+1}=v_{i k}+\Delta t \varphi_{i} S_{i} P_{i k}+\Delta t\left(q_{\mathrm{in} k}^{i}-q_{\mathrm{out}_{k}}^{i}\right)
$$

where $v_{i}$ corresponds to the volume in the $i$ th tank at time $k$ (given in cubic metres), $\varphi_{i}$ is the ground absorption coefficient' of the $i$ th catchment, $S$ is the surface area and $P_{k}$ is the rain intensity at each sample $k$. Flows $q_{\mathrm{in} k}^{i}$ and $q_{\text {out } k}^{i}$ are the sum of inflows and outflows, respectively. 'Real retention tanks, which correspond to the sewer network reservoirs, are modelled in the same way but without the precipitation term. Tanks are connected with flow paths or 


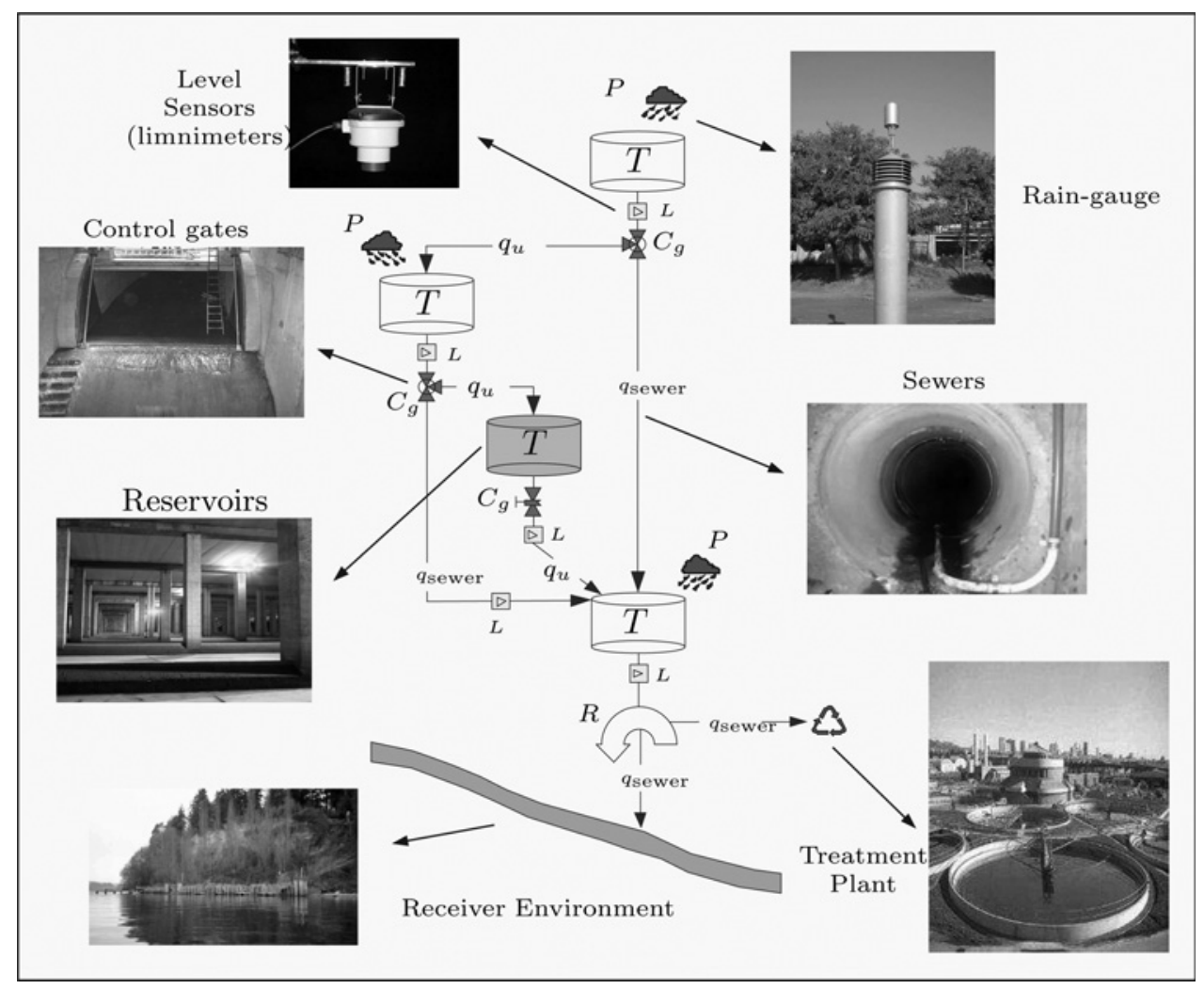

Figure 2 Simple sewer network composed of constitutive elements described in Section 2.2

links, which represent the main sewage pipes between the tanks. Manipulated variables of the system, denoted as $q_{u_{i}}$, are related to the outflows from the control gates. Tank outflows are assumed to be proportional to the tank volume, that is

$$
q_{\text {out } k}^{i}=\beta_{i} v_{i_{k}}
$$

where $\beta_{i}$ (given in $\mathrm{s}^{-1}$ ) is defined as the volume/flow conversion' coefficient as suggested in [17] by using the linear tank model approach. Notice that this relation can be made more accurate (but more complex) if (4) is considered to be non-linear (non-linear tank model approach). Limits on the volume range of real tanks are expressed as

$$
0 \leq v_{i_{k}} \leq \bar{v}_{i}
$$

where $\bar{v}_{i}$ denotes the maximum volume capacity given in cubic metres. As this constraint is physical, it is impossible to send more water to a real tank than it can store. Notice that real tanks without overflow capability have been considered. Virtual tanks do not have a physical upper limit on their capacity. When they rise above a pre-established volume, an overflow situation occurs. This fact represents the case when level in sewers has reached a limit so that an overflow situation can occur in the streets (flooding). Hence, when virtual tanks maximum volume $\bar{v}$ is reached, the excess volume above this maximum amount is redirected to another tank (catchment) within the network or to a receiver environment (as pollution). This situation creates a new flow path from the tank, denoted as $q_{d}$ (referred to as 'virtual tank overflow') that can be expressed mathematically as

$$
q_{d_{k}}= \begin{cases}\frac{\left(v_{k}-\bar{v}\right)}{\Delta t} & \text { if } \quad v_{k} \geq \bar{v} \\ 0 & \text { otherwise }\end{cases}
$$

In this case, the outflow of virtual tank is then limited by its maximum volume capacity as follows:

$$
q_{\text {out }_{k}}= \begin{cases}\beta \bar{v} & \text { if } v_{k} \geq \bar{v} \\ \beta v_{k} & \text { otherwise }\end{cases}
$$

Consequently, considering the tank overflow, the difference equation (3) in case of virtual tanks becomes

$$
v_{i_{k+1}}=v_{i_{k}}+\Delta t \varphi_{i} S_{i} P_{i_{k}}+\Delta t\left(q_{\mathrm{in} k^{i}}^{i}-q_{\mathrm{out}_{k}}-q_{d_{k}}\right)
$$

Using the proposed PWLF modelling approach, the tank outflows can be expressed as

$$
\begin{gathered}
q_{\text {out }_{k}}=\beta \operatorname{sat}\left(v_{k}, \bar{v}\right) \\
q_{d_{k}}=\frac{\operatorname{dzn}\left(v_{k}, \bar{v}\right)}{\Delta t}
\end{gathered}
$$

On the other hand, as noticed before, real tanks (see Fig. 1b) are elements designed to retain water in the case of intense rain. For this reason, both tank inflows and outflows are 
controlled. In the same way, tank inflow is constrained by the current volume within the real tank, by its maximum capacity and by the tank outflow. Since real tanks are considered without overflow capabilities, inflow is pre-manipulated by using a redirection gate (explained in Section 2.2.2), what leads to include the management policy in the model of the real tank. Proceeding in this way, the value of the manipulated flow $q_{a_{k}}^{\star}$ is restricted to the maximum flow condition in the input gate, and the flow through input link $q_{a}$ is expressed as

$$
\tilde{q}_{a_{k}}= \begin{cases}q_{a_{k}}^{\star} & \text { if } \quad q_{a_{k}}^{\star} \leq q_{\mathrm{in}_{k}} \\ q_{\mathrm{in}_{k}} & \text { otherwise }\end{cases}
$$

However, maximum tank capacity also constrains the inflow according to the expression

$$
q_{a_{k}}= \begin{cases}\tilde{q}_{a_{k}} & \text { if } \quad q_{b_{k}}-q_{\mathrm{out}_{k}} \leq \frac{\bar{v}-v_{k}}{\Delta t} \\ \frac{\bar{v}-v_{k}}{\Delta t} & \text { otherwise }\end{cases}
$$

Thus, the real tank outflow is given by

$$
q_{\text {out }_{k}}= \begin{cases}q_{\mathrm{out}_{k}}^{\star} & \text { if } \quad q_{\mathrm{out}_{k}}^{\star} \leq \beta v_{k} \\ \beta v_{k} & \text { otherwise }\end{cases}
$$

taking into account that $q_{\text {out }}^{\star}$ is also limited by the maximum capacity of the outflow link, denoted by $\bar{q}_{\text {out }_{k}}$, leading to the following difference equation:

$$
v_{k+1}=v_{k}+\Delta t\left(q_{a_{k}}-q_{\text {out }_{k}}\right)
$$

Notice that the flow through $q_{b}$ can be derived from the mass balance

$$
q_{b_{k}}=q_{\mathrm{in}_{k}}-q_{a k}
$$

The PWLF model for this element considering the tank inflow and outflow expressions is

$$
\begin{gathered}
q_{\text {out }_{k}}=\operatorname{sat}\left(q_{\text {out }_{k}}^{\star}, \beta v_{k}\right) \\
q_{a_{k}}=\operatorname{sat}\left(q_{a_{k}}^{\star}, \min \left(\frac{\bar{v}-v_{k}}{\Delta t}, q_{\mathrm{in}_{k}}\right)\right)
\end{gathered}
$$

2.2.2 Manipulated gates: Within a sewer network, gates are elements used as control devices since they can change the flow downstream. Depending on the action made, gates can be classified as 'redirection gates', used to change the direction of the sewage flow, and 'retention gates', used to retain the sewage flow in a certain network point (sewer or reservoir). In the case of real tanks, a retention gate is present to control the outflow. Virtual tank outflows cannot be closed but can be diverted using redirection gates. Indeed, redirection gates divert a flow from the nominal path which the flow follows if the gate is closed. This nominal flow is denoted as $Q_{i}$ in the equation below, which expresses the mass conservation relation in the element

$$
q_{\text {out } k}^{i}=Q_{i_{k}}+\sum_{j} q_{u_{i k}^{j}}^{j}
$$

where $j$ is an index over all manipulated flows coming from the gate. Fig. $1 c$ shows a conceptual scheme of redirection gates considered in this paper. Assuming that the flow through sewer $q_{a}$ is imposed (e.g. computed by means of a control law), the expressions that describe a redirection gate can be written as

$$
q_{a_{k}}= \begin{cases}\bar{q}_{a} & \text { if } q_{a}^{\star}>\bar{q}_{a} \\ q_{a}^{\star} & \text { otherwise }\end{cases}
$$

where $q_{a}^{\star}$ corresponds to the imposed/computed value for the flow $q_{a_{k}}$. Flow $q_{b_{k}}$ is directly given by the mass balance expression

$$
q_{b_{k}}=q_{\mathrm{in}_{k}}-q_{a_{k}}
$$

In the case of redirection gates, the PWLF model is defined taking into account that $q_{a}$ should satisfy the restriction (19), what can be rewritten in terms of the PWL functions as

$$
q_{a_{k}}=\operatorname{sat}\left(q_{a_{k}}, q_{\mathrm{in}_{k}}\right)
$$

Flow through $q_{b}$ is given by the mass balance (20).

2.2.3 Weirs and main sewer pipes: Since the description of their dynamics is very close, both types of elements are presented together in this section. 'Nodes' are points of the network where the sewage can be either propagated or merged. Hence, these elements can be classified as 'splitting nodes' and 'merging nodes'. The first type can be treated considering a constant partition of the sewage flow in predefined portions according to the topological design characteristics. Merging nodes exhibit a switching behaviour. In the case of a set of $n$ inflows $q_{i}$, with $i=1,2, \ldots, n$, the expression for the node outflow $q_{\text {out }}$ is written as

$$
q_{\text {out }}=\sum_{i=0}^{n} q_{i}
$$

'Weirs' can be seen as splitting nodes having a maximum capacity in the nominal outflow path related to the flow capacity of the output pipe. In the same way, 'main sewer pipes' can be seen as weirs with a single inflow. They are used as connection devices between network constitutive elements. Therefore considering the similarity between all the aforementioned elements and the notation in Fig. $1 d$, the set of expressions that represent the behaviour either of a weir or of a sewer pipe 
are the following:

$$
\begin{gathered}
q_{b_{k}}= \begin{cases}\bar{q}_{b} & \text { if } \quad q_{\text {in }}>\bar{q}_{b} \\
q_{\mathrm{in}_{k}} & \text { otherwise }\end{cases} \\
q_{c_{k}}= \begin{cases}q_{\mathrm{in}_{k}}-\bar{q}_{b} & \text { if } \quad q_{\text {in }}>\bar{q}_{b} \\
0 & \text { otherwise }\end{cases}
\end{gathered}
$$

where $\bar{q}_{b}$ is the maximum flow through $q_{b}$ and $q_{\text {in }}$ is the inflow. Notice that the outflow from virtual tanks is assumed to be unlimited in order to guarantee a feasible solution of an associated optimisation problem within the design procedure of a optimisation-based control strategy. The same idea applies to the outflow $q_{b_{k}}$ related to retention gates. However, most often, sewer pipes have limited flow capacity. When the limit of flow capacity is exceeded, the resultant overflow is possibly redirected to another element within the network or is considered as loss to the environment.

The PWLF model for main sewer pipes (or single inflow weirs) can be obtained from the overflow condition as follows:

$$
\begin{aligned}
& q_{b_{k}}=\operatorname{sat}\left(q_{\mathrm{in}_{k}}, \bar{q}_{b}\right) \\
& q_{c_{k}}=\operatorname{dzn}\left(q_{\mathrm{in}_{k}}, \bar{q}_{b}\right)
\end{aligned}
$$

where $\bar{q}_{b}$ corresponds again to the maximum flow capacity of the nominal outflow pipe.

\section{MPC-based RTC on large-scale sewer networks}

\subsection{MPC as a tool for global RTC}

In most sewer networks, the regulated elements (pumps, gates and retention tanks) are typically controlled locally, that is, they are controlled by a remote station according to the measurements of sensors connected only to that station. However, a global RTC system requires the use of an operational model of the network dynamics in order to compute, ahead of time, optimal control strategies for the network actuators based on the current state of the system [provided by supervisory control and data acquisition (SCADA) sensors], the current rain intensity measurements and appropriate rainfall predictions. The computation procedure of an optimal global control law should take into account all the physical and operational constraints of the sewage system, producing set-points which achieve minimum flooding and CSO.

As discussed in the introduction, MPC is a suitable control strategy to implement global RTC of sewer networks since it has some features to deal with complex systems such as sewer networks: big delays compensation, use of physical constraints, relatively simple for people without deep knowledge of control, multi-variable systems handling etc. Hence, according to [1], such controllers are very suitable to be used in the global control of urban drainage systems within a hierarchical control structure $[18,5]$. MPC, as the global control law, determines the set-points for local controllers of the sewer network. A management level is used to provide MPC with the operational objectives, what is reflected in the controller design as the performance indexes to be minimised. In the case of urban drainage systems, these indexes are usually related to flooding, pollution, control energy etc.

\subsection{MPC on sewer networks}

3.2.1 Control objectives: The sewage system control problem has multiple objectives with varying priority, see [5]. The type, number and priority of those objectives can also be different depending on the particular sewage system design. However, the most common objectives are generally related to the manipulation of the sewage in order to avoid undesired sewage flows outside of the main sewers (flooding). The main considered objectives for the case study presented in this paper are listed below in order of decreasing priority:

- Objective 1: minimise flooding in streets (virtual tank overflow).

- Objective 2: minimise flooding in links between virtual tanks.

- Objective 3: maximise sewage treatment.

A secondary purpose of the third objective is to reduce the volume in the tanks to anticipate future rainstorms. This objective also indirectly reduces pollution to the environment. This is because if the treatment plants are used optimally with the storage capacity of the network, pollution should be strongly minimised. Moreover, this objective can be complemented by conditioning minimum volume in real tanks at the end of the prediction horizon. It could be seen as a fourth objective.

3.2.2 Problem constraints: When using the modelling representations based on virtual tanks, either in MLD or PWLF form, only flow rates are manipulated in such way that some the inherent non-linearities (e.g. non-linear relation between gate opening and discharge flow) of the sewer network are simplified as discussed in [2]. However, in turn, some physical restrictions need to be included as constraints on system variables. For instance, variables $q_{u_{i}}^{j}$ that redirect outflow from a virtual tank should never be larger than the outflow from the tank. This is expressed with the following inequality:

$$
\sum_{j} q_{u_{i} k}^{j} \leq q_{\text {out } k}^{i}=\beta v_{i_{k}}
$$

Additionally, operational constraints associated with the range of gates actuation lead to the manipulated flows have to fulfill $q_{u_{i} k}^{j} \leq \bar{q}_{u_{i}}^{j}$, where $\bar{q}_{u_{i}}^{j}$ denotes its upper limit. 
Similarly, operational limits on the range of real tank volumes should be included (see (5)) to limit the amount of sewage that can be stored.

3.2.3 MPC disturbances: Rain plays the role of measured disturbance in the sewer networks MPC problem. The type of disturbance model to be used depends on the rain prediction procedure available. Existing methods range from the use of time series [19] to the sophisticated utilisation of meteorological radars [20]. According to [5], different assumptions can be done for the rain prediction when an optimal/predictive control law is used for the RTC of sewer networks. Results show that the assumption of constant rain over a short prediction horizon gives results that can be compared with the the case of considering known rain over the prediction horizon, confirming similar results reported in $[2,21]$.

\section{Case study description}

\subsection{Barcelona sewer network}

The city of Barcelona has a CSS of approximately $1697 \mathrm{~km}$ length in the municipal area plus $335 \mathrm{~km}$ in the metropolitan area, but only $514.43 \mathrm{~km}$ are considered as the main sewer network. Its storage capacity is about three million of cubic metres, which implies a dimension three times greater than other cities comparable to Barcelona. It is worth to notice that Barcelona has a population which is around 1.59 million inhabitants on a surface of $98 \mathrm{~km}^{2}$, approximately. This fact results in a very high density of population. Additionally, the yearly rainfall is not very high (600 mm/year), but it includes heavy storms (up to $90 \mathrm{~mm} / \mathrm{h}$ ) typical of the Mediterranean climate that can cause a lot of flooding problems and CSO to the receiving environments.

'Clavegueram de Barcelona, SA' (CLABSA) is the company in charge of the sewage system management in Barcelona. There is a remote control system in operation since 1994 which includes sensors, regulators, remote stations, communications and a control centre in CLABSA. Nowadays, as regulators, the urban drainage system contains 21 pumping stations, 36 gates, 10 valves and 8 retention tanks which are regulated in order to prevent flooding and $\mathrm{CSO}$. The remote control system is equipped with 56 remote stations including 23 rain-gauges and 136 water-level sensors which provide real-time information about rainfall and water levels into the sewage system. All this information is centralised at the CLABSA control centre through a SCADA system. The regulated elements (pumps, gates and retention tanks) are currently controlled locally, that is, they are handled from the remote control centre according to the measurements of sensors connected only to local stations.

\subsection{Barcelona test catchment}

This paper considers a portion of the Barcelona sewer network, which represents the main phenomena and the most common characteristics appeared in the entire network. This representative portion is selected to be the case study of this paper because a calibrated and validated model of the network obtained using the virtual modelling methodology (see Section 2) is available as well as rain gauge data for an interval of several years. The considered Barcelona test catchment (BTC) has a surface of $22.6 \mathrm{~km}^{2}$ and includes typical elements of the larger network.

The BTC has one retention gate associated with one real tank, three redirection gates and one retention gate, 11 sub-catchments defining equal number of virtual tanks, several level gauges (limnimetres) and two WWTPs. Also, there are five rain-gauges used to measure the rain entering in each sub-catchment. Notice that some sub-catchments (virtual tanks) share the same rain sensor. These sensors count the amount of tipping events in 5 min (sampling time) and such values is multiplied by $1.2 \mathrm{~mm} / \mathrm{h}$ in order to obtain the rain intensity $P$ in $\mathrm{m} / \mathrm{s}$ at each sampling time, after the appropriate units conversion. The difference between the rain inflows in virtual tanks that share the same rain gauges is because of the particular surface area $S_{i}$ and the ground absorption coefficient $\varphi_{i}$ of the corresponding subcatchment (see (3)), what yields to different amount of rain inflows.

Using the virtual tanks representation principle, the resultant BTC model has 12 state variables that represent the volumes of the 12 tanks ( 1 real and 11 virtual), 4 control inputs that correspond to the manipulated gate flows and 5 measured disturbances associated to the measurements of rain intensity at the sub-catchments. Two WWTPs are used to treat the sewage before it is released to the environment. The states related to the virtual tank volumes are estimated by using the limnimetres shown with capital letter L in Fig. 3. The free flows to the environment $\left(q_{10 \mathrm{M}}, q_{7 \mathrm{M}}, q_{8 \mathrm{M}}\right.$ and $q_{11 \mathrm{M}}$ to the Mediterranean sea and $q_{12 s}$ to other catchment) and the flows to the WWTPs $\left(q_{7 \mathrm{~L}}\right.$ and $\left.q_{11 \mathrm{~B}}\right)$ are also shown in the figure as well as rain intensities $P_{13}, P_{14}, P_{16}, P_{19}$ and $P_{20}$. The four manipulated flows, denoted as $q_{u_{i}}$, have a maximum capacity of $9.14,25$, 7 and $29.3 \mathrm{~m}^{3} / \mathrm{s}$, respectively. These limits cannot be relaxed since they are physical restrictions of the system (hard constraints).

\subsection{Rain episodes}

Rain episodes used for the simulation of the BTC and for the design of MPC strategies are based on real rain gauge data obtained within the city of Barcelona on the given dates (yyyy-mm-dd) as presented in Table 1. These episodes were selected because they represent the meteorological behaviour of Barcelona weather. Table 1 also shows the maximum 'return rate' among all five rain gauges in each episode. (The return rate - or period - is defined as the average interval of time within which a hydrological event of given magnitude is expected to be equaled or exceeded exactly 


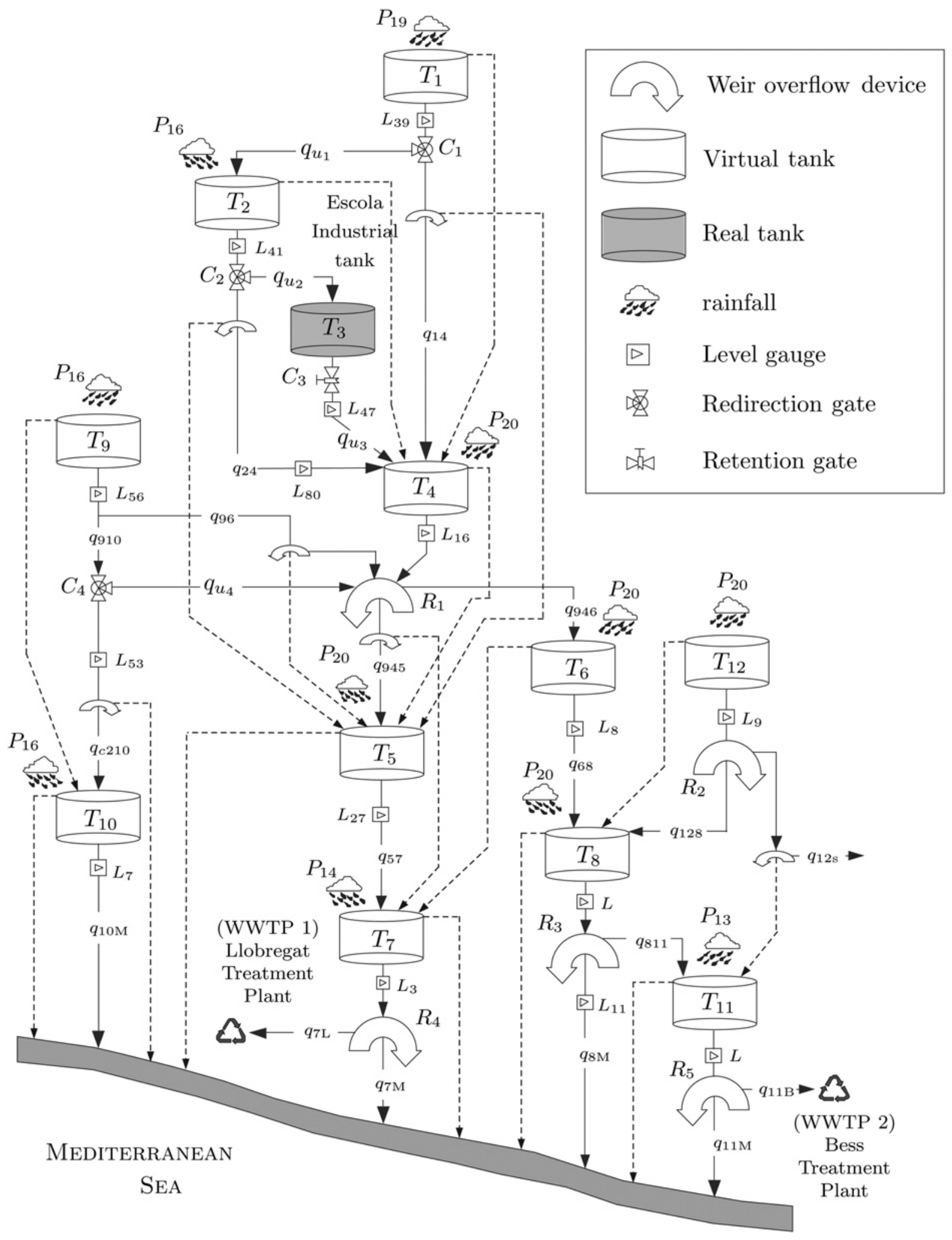

Figure 3 Barcelona test catchment scheme

once. In general, this amount is given in years.) In the third column of the table, the return rate for the whole Barcelona network is shown. Notice that one of the rain storms had a return rate of 4.3 years in the case of the whole network while for one of the rain gauges the return rate was 16.3 years. 


\section{Simulations and results}

\subsection{Preliminaries}

This section is focused on comparing the performance of the MPC-based sewer network RTC based on the PWLF-based modelling approach proposed in this paper with the hybrid MLD modelling approach suggested in [9] using a set of real rain episodes in Table 1 . Computation time, when both modelling approaches are used, is also compared. Results of such comparison would be a key issue to show the benefits of using the modelling approach proposed in this paper for RTC implementation in the real network case study. The assumptions made for all the implementations will be presented and their validity discussed before the results are given. The detailed description of BTC case study including operating ranges of the control signals and state variables as well as the description of all variables and parameters can be found in $[9,13]$.

Remark 1: In order to give an adequate idea of the complexity of the hybrid problem, system model in MLD form can be equivalently represented in PWA form as stated in [22]. Hence, the system can be expressed as

$$
\begin{aligned}
& x(k+1)=A_{i} x(k)+B_{i} u(k)+f_{i}, \quad i=1, \ldots, N_{d} \\
& \text { for }\left[\begin{array}{l}
x(k) \\
u(k)
\end{array}\right] \in \Omega_{i}
\end{aligned}
$$

where $x(k) \in \mathbb{R}^{n_{c}}$ is the vector of $n_{c}$ system states (network volumes at tanks), $u(k) \in \mathbb{R}^{m i_{c}}$ corresponds to the vector of the $m i_{c}$ control inputs (manipulated flows), $f_{i}$ are real vectors associated to the model disturbances (rain) and $N_{d}$ denotes the number of different dynamics.

According to [23], the way of obtaining the PWA system from the MLD form is based on the ennumeration of all $2^{r_{\ell}}$ combinations of binary variables, where $r_{\ell}$ is the number of auxiliary Boolean variables of the MLD model. In the case of the application shown in this paper, the MLD model considers $r_{\ell}=22$ (see [9] for further details), what leads in

Table 1 Rain episodes used for comparing modelling approaches

\begin{tabular}{|l|c|c|}
\hline Rain episode & $\begin{array}{c}\text { Maximum return } \\
\text { rate, years }\end{array}$ & $\begin{array}{c}\text { Return rate } \\
\text { average, years }\end{array}$ \\
\hline $1999-09-14$ & 16.3 & 4.3 \\
\hline $2002-07-31$ & 8.3 & 1.0 \\
\hline $2002-10-09$ & 2.8 & 0.6 \\
\hline $1999-10-17$ & 1.2 & 0.7 \\
\hline $2000-09-28$ & 1.1 & 0.4 \\
\hline
\end{tabular}

$N_{d}=4194304$ different dynamics. Since the complexity associated to the dimension of the PWA model is high enough, the alternative of using this representation for the MPC design was not considered.

\subsection{Simulation and prediction models}

Results presented in this paper have been obtained in simulation by using two different models: one used as the plant (sewer network), which in the sequel will be called as 'open-loop model', and the other used by the MPC controller, called 'control model'. The open-loop model is implemented considering a non-linear representation of the sewer network based on mass balances where ranges and bounds for every variable (control signals, volumes and rain disturbances) are strictly considered and all passible logical or discontinuous dynamics are included (as the case of weirs and overflows). On the other hand, the control model is obtained by using the modelling approach presented in Section 2. This model, considering the PWLF-based modelling approach, was obtained by joining the different compositional elements described in Section 2.2 and following the network diagram of Fig. 3, resulting in a non-linear representation as a set of expressions for the whole network.

\subsection{MPC controller set-up and implementation}

Different parameters of the MPC controller should be defined and tuned according to the control objectives and their prioritisation. According to the discussion in Section 3.2.1, to take into account the control objectives for the RTC of a sewer network, the following system outputs have been included in the control model

$$
\begin{gathered}
y_{1_{k}}=\sum_{i} q_{\mathrm{str}_{v} k}+\sum_{j} q_{\mathrm{str}_{q} k} \\
y_{2_{k}}=\sum_{l} q_{\mathrm{sea}_{k}} \\
y_{3_{k}}=q_{\operatorname{trp}_{k}}-\bar{q}_{7 \mathrm{~L}} \\
y_{4_{k}}=q_{\operatorname{trp}_{k}}-\bar{q}_{11 \mathrm{~B}}
\end{gathered}
$$

where $y_{1_{k}}$ represents the sum of the $i$ overflows to street from virtual tanks at time $k$, denoted by $q_{\mathrm{str}_{v} k}$, plus the sum of the $j$ overflows to street from links (main pipes) at time $k$, denoted by $q_{\mathrm{str}_{q} k}$. Output $y_{2_{k}}$ represents the sum of the loverflows that are released to the sea (as receiver environment) at time $k$, denoted as $q_{\text {sea }_{v} k}$ and finally $y_{3_{k}}$ and $y_{4_{k}}$ represent the difference at time $k$ between the flows towards the WWTPs, denoted by $q_{\operatorname{trp} 1_{k}}$ and $q_{\mathrm{trp} 2_{k}}$, and the maximum WWTPs inflows. For the case study of this paper, $q_{\operatorname{trp} 1_{k}}=q_{7 \mathrm{~L}_{k}}$ and $q_{\mathrm{trp} 1_{k}}=q_{11 \mathrm{~B}_{k}}$, with their maximum flows $\bar{q}_{7 \mathrm{~L}}$ and $\bar{q}_{11 \mathrm{~B}}$, respectively. 
Using the outputs (29), the multi-objective cost function for the BTC can be written as follows using the weighted approach technique

$$
J\left(u_{k}, x_{k}\right)=\sum_{i=1}^{H_{p}}\left\|\boldsymbol{y}_{k+i \mid k}\right\|_{Q}^{2}
$$

where $\boldsymbol{y}_{k+i \mid k}$ is the output vector at the instant $k+i$ with respect to time instant $k$ and $H_{p}$ denotes the prediction horizon set equal to 6 , which is equivalent to $30 \mathrm{~min}$ with a sampling time $\Delta t=300 \mathrm{~s}$. This selection was based on the reaction time of the system to disturbances. Another reason for this selection is that the constant rain prediction assumed in this paper becomes less reliable for larger horizons. The length of the simulation scenarios is 100 samples, what allows to see the influence of the peak of the rain (disturbance) from the selected rain episode over the dynamics of the network and also over the dynamic of the closed loop. $Q$ corresponds to a matrix containing the weights $w_{i}$, each one related to a control objective. Notice that the desired prioritisation of the control objectives is given by the values $w_{i}$ that, for this case, determines a $Q$ matrix of the following form:

$$
\boldsymbol{Q}=\operatorname{diag}\left\{w_{\mathrm{str}} \boldsymbol{I} w_{\mathrm{sea}} \boldsymbol{I} w_{\mathrm{trp} 1} \boldsymbol{I} w_{\mathrm{trp} 2} \boldsymbol{I}\right\}
$$

where $I$ corresponds to a identity matrix of suitable dimensions. Here, $w_{\text {str }}=1, w_{\text {sea }}=10^{-1}, w_{\text {trp } 1}=10^{-3}$ and $w_{\text {trp2 }}=10^{-3}$.

Notice that, considering the PWLF-based modelling approach, a non-linear optimisation problem is stated. By replacing the non-linear equality constraints (coming from the definition of the PWLF model) in the objective function (30), a new optimisation problem with PWLFbased objective function and bounded constraints is obtained. The selection of the algorithm to solve such problem was done after the evaluation of several solvers available on Tomlab $^{\circledR}$ (e.g. 'conSolve', 'nlpSolve', among others). The 'structured trust region' (STR) algorithm (see [24]) was finally chosen because it provides an 'acceptable' trade-off between system performance and computation time. If the global optimum is desired, the mentioned nonlinear algorithm should be combined with a bisection approach as suggested in [12].

On the other hand, the MLD hybrid model of the BTC, suggested in [13], is used for the comparisons presented below with the PWLF model. This MLD model has 22 logical variables and 44 auxiliary variables. Using this model, a hybrid MPC controller has been designed and the set of considered rain scenarios were simulated using the 'Hybrid Toolbox' for Matlab ${ }^{\circledR}$ (see [25]) and ILOG CPLEX 11.2. This latter solver allows to handle efficiently the mixed-integer programming (MIP) problems associated to the hybrid MPC controller.

\subsection{Control performance and computation time comparisons}

For performance comparison purposes and additionally to the control results when the considered modelling approaches (hybrid and PWLF models) are used, results obtained when the network is in open loop are also presented. The computation times reported in this paper has been obtained using $\mathrm{Matlab}^{\circledR} 7.2$ implementations running on an Intel ${ }^{\circledR}$ Core $^{\mathrm{TM}}$ 2, $2.4 \mathrm{GHz}$ machine with $4 \mathrm{~Gb}$ RAM. Notice that computation time results reported here related with hybrid models are different from those presented in [13] because of the machine characteristics and solver versions.

The open-loop case consists in the sewage system without control so the manipulated links are used as passive elements, that is, the amount of the flows $q_{u 1}, q_{u 2}$ and $q_{u 4}$ only depend on the inflow to the corresponding gate and they are not manipulated while $q_{u 3}$ is the outflow of the real tank given by gravity (tank discharge). Results related to the control performance are summarised in Tables 2-4 for five representative rain episodes in Barcelona between 1998 and 2002 (yyyy-mm-dd in tables) presented in Table 1. Table 2 shows the comparison of volumes of sewage released to the street (flooding) while Table 3 shows the same comparison but regarding the volumes to receiver environments (pollution). Finally, Table 4 shows the comparison of volumes related to the treated sewage at the WWTPs.

Notice from Tables 2-4 that the performance of the system is better when an MPC control law is considered no matter the

Table 2 Performance results (flooding $\left(\times 10^{3} \mathrm{~m}^{3}\right)$ )

\begin{tabular}{|l|c|c|c|}
\hline Rain episodes & Open loop & PWLF model & Hybrid model \\
\hline $1999-09-14$ & 108 & 88.2 & 92.9 \\
\hline $2002-10-09$ & 116.1 & 113.3 & 97 \\
\hline $2002-07-31$ & 160.3 & 132.8 & 139.7 \\
\hline $1999-10-17$ & 0 & 0 & 0 \\
\hline $2000-09-28$ & 1 & 1 & 1 \\
\hline
\end{tabular}

Table 3 Performance results (pollution $\left(\times 10^{3} \mathrm{~m}^{3}\right)$ )

\begin{tabular}{|c|c|c|c|}
\hline $\begin{array}{c}\text { Rain } \\
\text { episodes }\end{array}$ & $\begin{array}{c}\text { Open } \\
\text { loop }\end{array}$ & PWLF model & $\begin{array}{c}\text { Hybrid } \\
\text { model }\end{array}$ \\
\hline $1999-09-14$ & 225.8 & $226.1(1.16 \%)$ & 223.5 \\
\hline $2002-10-09$ & 409.8 & $407.7(2.25 \%)$ & 398.7 \\
\hline $2002-07-31$ & 378 & $380(1.44 \%)$ & 374.6 \\
\hline $1999-10-17$ & 65 & $59.9(3.09 \%)$ & 58.1 \\
\hline $2000-09-28$ & 104.5 & $102(4.08 \%)$ & 98 \\
\hline
\end{tabular}


Table 4 Performance results (treated sewage at WWTPs $\left.\left(\times 10^{3} \mathrm{~m}^{3}\right)\right)$

\begin{tabular}{|c|c|c|c|}
\hline $\begin{array}{c}\text { Rain } \\
\text { episodes }\end{array}$ & $\begin{array}{c}\text { Open } \\
\text { loop }\end{array}$ & PWLF model & $\begin{array}{c}\text { Hybrid } \\
\text { model }\end{array}$ \\
\hline $1999-09-14$ & 278.3 & $276.7(1.43 \%)$ & 280.7 \\
\hline $2002-10-09$ & 533.8 & $534.2(1.98 \%)$ & 545 \\
\hline $2002-07-31$ & 324.3 & $321.9(1.80 \%)$ & 327.8 \\
\hline $1999-10-17$ & 288.4 & $293.5(0.61 \%)$ & 295.3 \\
\hline $2000-09-28$ & 285.3 & $287.5(1.51 \%)$ & 291.9 \\
\hline
\end{tabular}

modelling approach utilised with respect to the performance in open loop. This justifies the use of closed-loop control. Moreover, notice also that the use of the hybrid modelling approach implies in average a better system performance with respect to the performance improvement obtained by using the PWLF-based modelling approach. Notice also that the performance improvement is basically related to the improvement of the main control objective, and then following in a hierarchical order, to the second objective and so on. In this way, notice that the pollution for some episodes is worse with respect to the open-loop case - see, for example, Table 3, episodes 1999-09-14 and 2002-07-31. However, notice that the performance index associated to the flooding is the best for both episodes, following the preestablished control objectives priority.

In general, these results were expected since the MPC controller based on the hybrid modelling approach achieves its optimum by solving a set of convex linear problems using a branch and bound scheme. However, the MPC based on PWLF-based modelling approach leads to a nonlinear network model representation what might result in a quasi-convex optimisation. Therefore using the STR algorithm, the global optimum cannot be assured because bisection method was not implemented in this paper. This fact leads possibly to a sequence of suboptimal control actions when the computation of the RHC law is done. This explains why the performance obtained using the PWLF model is in general worse than the one obtained using the hybrid model. Suboptimality levels of the results obtained using the PWLF model were never greater that $4.1 \%$ for the cases of the second and third objective (as shown in Tables 3 and 4 in parenthesis). For the case of the first control objective (related to flooding), results were not so homogeneous since for some scenarios one of the modelling approaches leads in better system performance while for other scenarios occurred just the opposite.

On the other hand, the main difference of using the hybrid or the PWLF modelling approaches is in the computation time required to determine the control actions at each iteration. As mentioned in Section 5.2, the model in MLD form contains an important number of Boolean and auxiliary variables. In the BTC case study, to determine the control actions using hybrid MPC implies that for each time instant, considering a prediction horizon $H_{\mathrm{p}}=6$, $2^{22 \times 6}=5.4 \times 10^{39}$ linear programming (LP) problems (for a linear norm in the cost function) or quadratic programming (QP) problems (for a quadratic norm in the cost function) should be solved in the worst case. Thus, the complexity of the MIP associated to the MPC law becomes bigger by increasing the number of Boolean variables since the underlying optimisation problem is combinatorial and $\mathcal{N P}$-hard [26]. Thus, the worst-case computation time is exponential in the amount of integer variables. In large-scale systems such as sewer networks, the amount of elements with logical/discontinuous dynamics can augment according to the topology of the particular case study. Therefore computation times increase towards a point where the use of this modelling for obtaining an MPC-based RTC law becomes almost impossible. On the other hand, the alternative modelling approach based on the PWLFs proposed on this paper allows to have control sequences computed in lower times at the price of some degree of suboptimality because of the possible local optimum. Table 5 summarises the computation times for both the modelling approaches proposed on this paper and for the five rain episodes previously considered. It can be seen for the last two rain episodes that the computation times for both modelling approaches are almost equivalent. According to [9], this difference relies on the influence of the system disturbances within the optimisation problem. Depending on the variability of the rain and its intensity in function of the location, more discontinuous behaviours might be observed. This fact can determine the relative complexity of the MIP problem but it does not influence in the same way the optimisation problem based on PWLF models. When a hybrid model is used, depending on the evolution of the rain intensity, many modes can be changed and the global behaviour of the system represented by the hybrid model becomes more complex (e.g. new flows can

Table 5 Computation time results $(\mathrm{s})$

\begin{tabular}{|l|c|c|c|c|}
\hline \multirow{2}{*}{$\begin{array}{l}\text { Rain } \\
\text { episodes }\end{array}$} & \multicolumn{2}{|c|}{ PWLF model } & \multicolumn{2}{c|}{ Hybrid model } \\
\cline { 2 - 5 } & $\begin{array}{c}\text { Total } \\
\text { CPU } \\
\text { time }\end{array}$ & $\begin{array}{c}\text { Maximum } \\
\text { CPU time in } \\
\text { a sample }\end{array}$ & $\begin{array}{c}\text { Total } \\
\text { CPU } \\
\text { time }\end{array}$ & $\begin{array}{c}\text { Maximum } \\
\text { CPU time in } \\
\text { a sample }\end{array}$ \\
\hline $\begin{array}{l}1999-09- \\
14\end{array}$ & 695.33 & 91.32 & 1109.29 & 787.17 \\
\hline $\begin{array}{l}2002-10- \\
09\end{array}$ & 293.23 & 66.01 & 561.73 & 85.31 \\
\hline $\begin{array}{l}2002-07- \\
31\end{array}$ & 830.20 & 83.04 & 1050.54 & 381.49 \\
\hline $\begin{array}{l}1999-10- \\
17\end{array}$ & 180.22 & 16.15 & 79.14 & 10.39 \\
\hline $\begin{array}{l}2000-09- \\
28\end{array}$ & 120.88 & 12.13 & 84.76 & 13.27 \\
\hline
\end{tabular}


appear, Hamming distance between the Boolean variables vectors in actual time instant and the previous step gets bigger etc.). This fact makes that the mixed-integer optimisation gets also complex and requires high computational time. On the other hand, when a PWLFbased model is used under the same rain intensity conditions, despite the optimisation is non-linear, the computation does not depend on Boolean variables (which determine the current mode of the system in the hybrid model) and the computational time gets lower.

Summarising, although the suboptimal nature of the solutions as a consequence the minor improvement of the control performance, the MPC controller considering the PWLF-based modelling approach not only leads to a faster control sequences computation but also to feasible ones with respect to the real-time restriction imposed by the sampling time. In average, all the maximum computation times to compute the MPC control action when the PWLF-based modelling approach is used are less than the third part of the sampling time. This is not the case when using the hybrid modelling approach.

\section{Conclusions}

In this paper, RHC of large-scale sewage systems has been addressed considering a modelling approach based on PWLF. This modelling approach is compared against a hybrid modelling approach previously reported by the authors within the RHC framework. Control performance results and associated computation times of both approaches were compared by using a real case study based on the Barcelona sewer network. With the PWLF-based modelling formulation proposed, although a small amount of suboptimality is introduced since the resultant non-linear optimisation problem is non-convex, the reduction in the computation time allows to face the control of large-scale sewer networks. The future work is already focused on the quasi-convexity theoretical proof of optimisation problem based on PWLF models in order to take advantage of the modelling using a family of quasi-convex functions. This way would allow to find solutions to the optimisation problem with a lower level of suboptimality with respect to the solutions found for a pure non-linear optimisation problem.

\section{Acknowledgments}

This work has been supported by the CICYT WATMAN (ref. DPI2009-13744) of the Spanish Science and Technology Ministry, the 'Juan de la' Cierva Research Programme (ref. JCI-2008-2438), and the DGR of Generalitat de Catalunya (SAC group, ref. 2009/SGR/1491).

\section{References}

[1] SCHÜTZE M., CAMPISANOB A., COLAS H., SCHILLINGD W., VANROLLEGHEM P.: 'Real time control of urban wastewater systems: where do we stand today?', J. Hydrol., 2004, 299, pp. 335-348

[2] GELORMINO M., RICKER N.: 'Model predictive control of a combined sewer system', Int. J. Control, 2009, 59, pp. $793-816$

[3] pleau M., methot F., lebrun A., colas A.: 'Minimizing combined sewer overflow in real-time control applications', Water Qual. Res. J. Canada, 1996, 31, (4), pp. $775-786$

[4] pleau M., colas h., lavalle P., Pelletier G., bonin R.: 'Global optimal real-time control of the Quebec urban drainage system', Environ. Model. Softw., 2005, 20, pp. 401-413

[5] MARINAKI M., PAPAGEORGIOU M.: 'Optimal real-time control of sewer networks' (Springer, 2005)

[6] DUCHeSNe S., MAilhot A., DEQUidT E., VIlleneuve J.: 'Mathematical modeling of sewers under surcharge for real time control of combined sewer overflows', Urban Water, 2001, 3, pp. 241-252

[7] OCAMPO-MARTINEZ C., INGIMUNDARSON A., PUIG V., QUEVEDO J.: 'Objective prioritization using lexicographic minimizers for MPC of sewer networks', IEEE Trans. Control Syst. Technol., 2008, 16, (1), pp. 113-121

[8] Cembrano G., QUeVedo J., SAlAMERo M., PUig V., figueras J., MARTí J.: 'Optimal control of urban drainage systems: a case study', Control Eng. Pract., 2004, 12, (1), pp. 1-9

[9] OCAMPO-MARTINEZ C., BEMPORAD A., INGIMUNDARSON A., PUIG V.: 'On hybrid model predictive control of sewer networks' in SANCHEZ-PEÑA R., PUIG V., QUEVEDO J. (EDS.): 'Identification and control: the gap between theory and practice' (Springer-Verlag, 2007)

[10] BEMPORAD A., MORARI M.: 'Control of systems integrating logic, dynamics, and constraints', Automatica, 1999, 35, (3), pp. 407-427

[11] SCHECHTER M.: 'Polyhedral functions and multiparametric linear programming', J. Opt. Theory Appl., 1987, 53, pp. 269-280

[12] Boyd S., VAndenberghe L.: 'Convex optimization' (Cambridge University Press, 2004)

[13] OCAMPO-MARTINEZ C.: 'Model predictive control of complex systems including fault tolerance capabilities: application to sewer networks', PhD thesis, Technical University of Catalonia, April 2007

[14] MAYS L.W.: 'Urban stormwater management tools' (McGraw-Hill, 2004) 
[15] MARINAKI M., PAPAGEORGIOU M.: 'Nonlinear optimal flow control for sewer networks' (Philadelphia, Pennsylvania, USA, 1998), vol. 2, pp. 1289-1293

[16] ERMOLIN Y.: 'Mathematical modelling for optimized control of Moscow's sewer network', Appl. Math. Model., 1999, 23, pp. $543-556$

[17] SINGH V.P.: 'Hydrologic systems: rainfall-runoff modeling' (Prentice-Hall, NJ, 1988), vol. 1

[18] PAPAgEORgIOU M.: 'Optimal multireservoir network control by the discrete maximum principle', Water Resour. Res., 1985, 21, (12), pp. 1824-1830

[19] SMITH K.T., AUSTIN G.L.: 'Nowcasting precipitation - a proposal for a way forward', J. Hydrol., 2000, 239, pp. 34-45

[20] YUAN J.M., TILFORD K.A., JIANG H.Y., CLUCKIE I.D.: 'Real-time urban drainage system modelling using weather radar rainfall data', Phys. Chem. Earth (B), 1999, 24, pp. 915-919

[21] OCAMPO-MARTINEZ C., PUIG V., QUEVEDO J., INGIMUNDARSON A.: 'Fault tolerant model predictive control applied on the
Barcelona sewer network'. Proc. IEEE Conf. on Decision and Control (CDC) and European Control Conf. (ECC), Seville, Spain, 2005

[22] HEEMELS W.P.M.H., DE SCHUTTER B., BEMPORAD A.: 'Equivalence of hybrid dynamical systems', Automatica, 2001, 37, (7), pp. 1085-1091

[23] Bemporad A.: 'Efficient conversion of mixed logical dynamical systems into an equivalent piecewise affine form', IEEE Trans. Automatic Control, 2004, 49, (5), pp. $832-838$

[24] CONN A.R., GOULD N., SARTENAER A., TOINT PH.L.: 'Convergence properties of minimization algorithms for convex constraints using a structured trust region', SIAM J. Opt., 1995, 6, (4), pp. 1059-1086

[25] BeMPORAD A.: 'Hybrid toolbox - user's guide', April 2006

[26] PAPADIMITRIOU C.: 'Computational complexity' (AddisonWesley, 1994) 